Proc. Indian Acad. Sci. (Chem. Sci.), Vol. 98. No: 3. March 1987, pp. 235-24). (C) Printed in India.

\title{
The role of solvent models in stabilizing nonclassical ions
}

\section{SATHEESAN BABU and BHALACHANDRA L TEMBE* $\dagger$}

School of Chemistry, University of Hyderabad, Central University P. O, Hyderabad 500134 India

$\dagger$ Present address: Department of Chemistry, Indian Institute of ${ }^{7}$ 'hnology, Powai, Bombay 400076 , India

MS received 20 December 1986; revised 12 March 1987

\begin{abstract}
A molecular dynamics simulation is performed for a system of classical and nonclassical 2-norbornyl cations surrounded by a model solvent. Comparison of the energies of stabilization of the two ions due to the model solvent medium indicates that the difference in stabilization energies of the two ions is less than $1 \mathrm{kcal} / \mathrm{mol}$.
\end{abstract}

Keywords. Molecular dynamics; classical and nonclassical ion controversy.

\section{Introduction}

To account for the difference between the exo and endo solvolysis of 2-norbornyl derivatives the nonclassical 2-norbornyl cation was postulated (see figure 1) (Winstein and Trifan 1952). The exo solvolytic rates were larger than the endo rates by factors of the order of 1000 (Goering and Schewene 1965). These rate differences lead to a difference in the activation free energies $(\triangle G)$ for the two reactions; the exo solvolysis having a $\triangle G$ around $6 \mathrm{kcal} / \mathrm{mol}$ smaller than the endo.
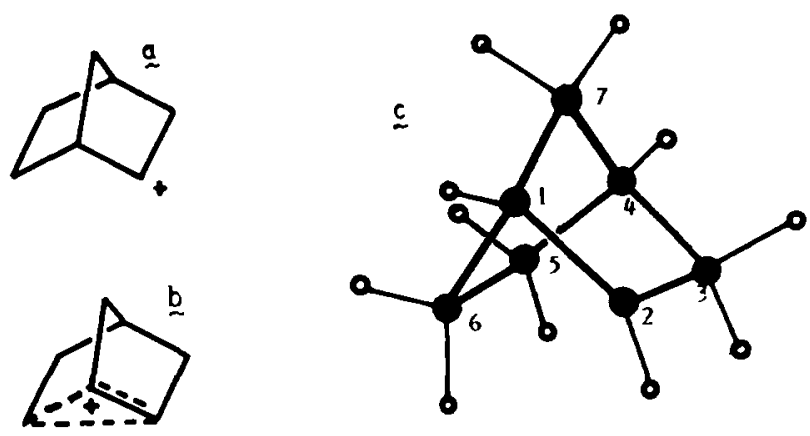

Figure 1. (a) Classical ion; (b) nonclassical ion; (c) geometry of classical ion: Bond lengths $(\AA) \mathrm{C} 1 \mathrm{C} 2(1.50), \mathrm{C} 1 \mathrm{C} 6(1.550), \mathrm{C} 1 \mathrm{C} 7(1.54), \mathrm{C} 6 \mathrm{C} 5(1.54), \mathrm{C} 2 \mathrm{C} 3(1.50), \mathrm{C} 5 \mathrm{C} 4$ $(1.55), \mathrm{C} 3 \mathrm{C} 4(1.556), \mathrm{C} 4 \mathrm{C} 7(1.54)$. Angles (degrees) $\mathrm{C} 1-\mathrm{C} 7-\mathrm{C} 4(96 \cdot 9), \mathrm{C} 7-\mathrm{C} 4-\mathrm{C} 3$ $(101 \cdot 4), \mathrm{C} 7-\mathrm{C} 4-\mathrm{C} 5(102 \cdot 4), \mathrm{C} 5-\mathrm{C} 4-\mathrm{C} 3(105 \cdot 4), \mathrm{C} 7-\mathrm{C} 1-\mathrm{C} 2$ (107.3). C7-C1-C6 (99.1), C6-C1-C2 (99.6), C4-C3-C2 (100-5), C4-C5-C6 (102.5), C1-C2-C3 (109.9), C1-C6-C5 $(102 \cdot 8)$. All other $\mathrm{C}-\mathrm{H}$ lengths and $\mathrm{H}-\mathrm{C}-\mathrm{H}$ angles are same as in Goddard et al 1982. 
In order to account for this difference, several $a b$ initio as well as semi-empirical quantum chemical calculations have been performed on the two isolated ions in the gas phase to see which one is more stable (Klopman 1969; Dewar et al 1977; Goetz et al 1977; Wenke and Lenoir 1979; Kohler and Lischka 1979; Goddard et al 1982; Raghavachari et al 1983; Yoshimine et al 1983). There is a considerable spread in the results obtained in these studies, no doubt due to the different levels of sophistication used in various calculations. In the above calculations the role of the solvent is not taken into account. To get an idea of the influence of the solvent, models of classical and nonclassical ethyl cations were surrounded by $5 \mathrm{HCl}$ molecules and the classical ion was found to be preferentially stabilized by $16 \mathrm{kcal} / \mathrm{mole}$ (Jorgensen and Munroe 1977). The existence of a nonclassical structure for norbornyl cation was confirmed earlier by Olah and coworkers (for a review, see Olah et al 1983) and recently by Yannoni et al (1982) and Myhre et al (1985) by spectroscopic measurements. However, Brown $(1983,1986)$ questions the arguments supporting the nonclassical structure based on various experimental factors and Schleyer has critically examined Brown's arguments (Brown 1977.). In the study of the solvation of the 2-norbornyl cation one has to have detailed knowledge of the nature of the interactions between the ion and the solvent molecules, and also among the solvent molecules themselves, and perform a statistical averaging over all possible solvent configurations around the ions. If the solvent molecules are dipoles or charged species, they will contribute significantly to ionic solvation. One may also like to consider the contribution from different solvation shells around the ions to the energy of solvation.

In a Monte Carlo simulation of the relative solvation of parallel and perpendicular allyl cations in a strongly hydrogen-bonding solvent (liquid HF), Jorgensen and coworkers found that the parallel allyl cation is strongly hydrogenbonded to two HF molecules (Cournoyer and Jorgensen 1984). The solvent contribution to stabilization was greater in the case of the perpendicular form than in the parallel form by $25 \mathrm{kcal} / \mathrm{mol}$. The potential functions used in the calculations were obtained by $a b$ initio calculations using a 4-31G basis set. Recent semi-empirical quantum calculations support the suggestion that the 2-norbornyl cation is a "nonclassical-classical" carbo cation (Dewar and Merz 1986). In our present work, we are exploring the changes in the solvation energies due to bridging, which has been postulated for the nonclassical 2-norbornyl cation. The model potentials and the method of calculation are discussed in the next section. This is followed by results and discussion.

\section{Method}

Our model represents the ions in a weakly ionic/dipolar low density solvent. The system is contained in a spherical cell of radius $8 \AA$ with the cation (solute) held fixed at the centre of the cell (Tembe and McCammon 1984). The coordinates of the ions are based on the data of Chiang et al (1968) and Goddard et al (1982). We have focussed our attention on the charge on the second carbon (C2), which is localized in the classical case and delocalized in the nonclassical case. In the nonclassical ion the only change that is made is in the distribution of the excess charge. The ions are surrounded by 28 model solvent particles. The system density 
is about half of the density at closest packing.

The interaction potential for particles $i$ and $j$ separated by a distance $r$ is given by

$$
U_{i j}(r)=4 \varepsilon\left[(\sigma / r)^{12}-(\sigma / r)^{6}\right]+q_{i} q_{j} / r,
$$

where the first term is the Lennard-Jones potential function with the parameters $\varepsilon / k=120 \mathrm{~K}$ and $\sigma=3.4 \mathrm{~A}$ representing the interaction between spherical particles at locations $i$ and $j$, and $q_{i}$ and $q_{j}$ the charges on particles $i$ and $j$, respectively. The actual interaction potential between the norbornyl ion and solvent are not available and even the interactions between simple ions like $\mathrm{Li}^{+}$and $\mathrm{Na}^{+}$with polar solvents like water are becoming available only in the last few years (Rossky 1985). In this work we are taking into account the dominant interactions between the ion and the solvent by the most common empirical potentials. The charge-charge interactions used here are on the average greater than the charge dipole interactions which decay as $1 / r^{3}$ and our results could be slight overestimates of the solvent contribution to stabilization. In simulation A the value of $\sigma$ was the same between all pairs of atoms. In simulation B the value of $\sigma$ was $3.4 \AA$ for all pairs except for the solvent and the hydrogen atoms on the solute ion. This latter value of $\sigma$ was taken to be $2.07 \AA$. The solvent particles have charges of $\pm 0 \cdot 1 e$, where $e$ is the electronic charge. This is equivalent to a charge of $1 e$ on $\mathrm{C} 2$ in a medium of dielectric constant of 10 . The $\mathrm{C}$ and $\mathrm{H}$ atoms of the cation interact with the solvent particles only via the Lennard-Jones interaction except that in case of the classical cation the centre $\mathrm{C} 2$ has a charge of $q_{2}=0.1 e$ and the electrostatic part of the interaction is given by

$$
U_{s}^{C}=\sum_{s=1}^{28} q_{2} q_{s} / r_{2 s}
$$

where $q_{s}$ is the charge on the solvent particle and $r_{2 s}$ is the distance between $\mathrm{C} 2$ and the solvent particle $s$. In the nonclassical ion the positive charge at $\mathrm{C} 2$ is distributed on a triangle formed between carbons 1,2 , and 6 . We have distributed 18 point charges on the triangle such that the total charge is the same as the charge on the classical cation $\left(q_{2}=0 \cdot 1 e\right)$. On each of the three bonds $\mathrm{C} 1 \mathrm{C} 2, \mathrm{C} 2 \mathrm{C} 6$ and $\mathrm{C} 6 \mathrm{C} 1$, one third of $q_{2}$ is spread uniformly. The electrostatic interaction with the solvent of the nonclassical ion is

$$
U_{s}^{N}=\sum_{s=1}^{28} \sum_{i=1}^{18} q_{n} q_{s} / r_{i s}
$$

where $q_{n}$ is the charge on each of the 18 points on the triangle, $r_{i s}$ the distance between point $i$ and the solvent particle $s$.

Using the potentials given above a molecular dynamics simulation is performed for the calculation of the energy of stabilization of the 2-norbornyl cation due to the solvent medium. Knowing the forces at each particle, new positions are calculated. This is achieved by numerically solving Newton's equations of motion. The time step for the simulation is $\mathbf{0 . 0 2}$ picoseconds. Stochastic boundary conditions are used wherein a uniform temperature is maintained by randomly reassigning velocities from a Maxwellian distribution at $298 \mathrm{~K}$ to 5 particles per time step. The particles are confined within the $8 \AA$ cell by imposing a harmonic wall potential. We use the Verlet algorithm to integrate the equations of motion (Verlet 1967). In our present 
studies we have focussed our attention on solute-solvent interaction energies. The system is allowed to equilibrate from a lattice configuration for the first 2000 steps of simulation. The average values of the solvent-solute and solvent-solvent interaction energies are calculated for the next 10,000 steps of simulation.

\section{Results and discussion}

In table 1 the results of our simulation are given. It is noticed that the difference in the solute-solvent interaction energies between the classical and nonclassical systems in simulation $A$ is $0.15 \mathrm{kcal} / \mathrm{mol}$. and in $B$ it is $0.4 \mathrm{kcal} / \mathrm{mol}$. As we change the closest distance of approach of the solvent particles up to the hydrogens of the two ions from $3.4 \AA$ to $2.07 \AA$, the stabilization has changed by 0.15 to $0.4 \mathrm{kcal} / \mathrm{mol}$. This implies that the small differences in $\mathrm{C}-\mathrm{C}$ and $\mathrm{C}-\mathrm{H}$ bond lengths between the classical and nonclassical ions (which are less than $2 \%$ ) are going to make a significant difference in our results for the stabilization due to solvation. Thus these model calculations show that the contribution of the solvent to the preferential stabilization of either of the ions after statistical averaging is less than $1 \mathrm{kcal} / \mathrm{mol}$. We are presently looking into the effect of system size as well as system density on the relative stabilization energies reported here. In a recent simulation by Engstrom et al (1984) it has been observed that the system size has a relatively small effect on the quadrupole relaxation of atomic ions. The simulation reported by us is analogous to a simulation where a charge of $1 e$ is localized on $\mathrm{C} 2$ or spread around $\mathrm{C} 2$ in a medium of dielectric constant of 10 but a density smaller than the actual density used in experimental systems. We are investigating the influence of the variation of $q_{2}$ and the dielectric constant reported here but we do not expect the preferential stabilization due to bridging to be greater than the experimentally observed differences in $\Delta G$ in media with dielectric constants greater than 10 .

Table 1. Average solvent-solvent and solute-solvent interaction energies in classical and nonclassical systems over $10,0(0)$ steps of simulation (20) ps).

Simulation A

\begin{tabular}{lccc}
\hline & Solvent-solvent & Solute-solvent & Total \\
\hline $\begin{array}{l}\text { Classical ion } \\
\begin{array}{l}\text { Nonclassical ion } \\
\text { Difference } \\
\text { (nonclassical-classical) }\end{array}\end{array}$ & -1.104 & -11.900 & -13.004 \\
$\begin{array}{l}\text { Simulation } B \\
\text { Slassical ion }\end{array}$ & 0.007 & -11.758 & -12.855 \\
$\begin{array}{l}\text { Nonclassical ion } \\
\text { Difference } \\
\text { (nonclassical-classical) }\end{array}$ & -1.158 & 0.142 & 0.149 \\
\hline
\end{tabular}

All computations were done using an IBM PC-XT; the classical ion took 15 minutes while the nonclassical ion 17 minutes for 100 steps of simulation. (All values in $\mathrm{kcal} / \mathrm{mole}$ ). 
Our calculations indicate a $\Delta E$ of about $1 \mathrm{kcal} / \mathrm{mol}$. in a non-hydrogen-bonding model solvent in contrast to Jorgensen's result of $25 \mathrm{kcal} / \mathrm{mol}$ for parallel and perpendicular allyl cations in liquid HF. The relative solvation of structures like parallel and perpendicular allyl cations and classical and nonclassical ethyl cations in our model solvent and models for solvents such as water, alcohol and $30 \%$ acetone will give us a clearer indication of the role of intermolecular interactions in solvation. The main route at present for obtaining the solute-solvent interactron energies is by the use of the molecular orbital method. Although there is no direct experimental method to verify these potentials in the condensed medium (as against the gas phase), results derived from these potentials will provide a quantitative answer to the relative magnitudes of solvation and also the detailed solvent particle distributions about the ions. As the solvation energies are derived from kinetic data it would be of great interest to investigate the structure and dynamics of the solvent particles when a leaving group such as tosylate or a halide ion is in the process of leaving from the exo and the endo positions. Calculations of potential energy surfaces between the ions and the solvent particles in a bulk medium or a condensed environment are being investigated quite actively by several investigators.

It may be of interest to compare the results obtained in this study with the results known for the electrostatic contribution to the solvation free energies of a spherical non-electrolyte solute molecule. The Born equation (Born 1920) for the solvation energy in a continuum dielectric is

$$
G_{\text {solv }}^{o}(i)=\left[N\left(Z_{i} e\right)^{2} / 2 r_{i}\right] /(1-1 / D) \text {. }
$$

In this expression $r_{i}$ is the radius of the ion, $Z_{i}$ is the valence of the ion, $e$ the electronic charge, $D$ the dielectric constant and $N$ the Avagadro number. As the radius of the ion is decreased, the solvation energy increases. As the charges get confined to smaller volumes such as edges and pointed tips, the electric field around them increases dramatically and the medium around them is polarized further resulting in larger solvation energy. Thus when we go from classical to nonclassical ions, the solvation energy is expected to decrease due to a greater spread from the original point or spherical charge. In the problem studied in our model, let us suppose that the charge contained at $\mathrm{C} 2$ of the classical ion of radius $r_{i}$ is distributed into 18 separated spheres of smaller radii such that the total volume of the droplets is equal to the original volume of the sphere. If we assume that these spherical droplets are independent, then the ratio of solvation energy of the classical to the nonclassical is given by $18^{2 / 3}$. In addition to the neglect of nonelectrostatic effects and the molecular nature of the solvent in the Born model, this calculation assumes that the charges in the nonclassical ion are separated beyond molecular distances. However, this gives an indication of the order of magnitude of results in a continuum model.

\section{Acknowledgements}

We thank Dr E D Jemmis for discussions and the UGC special assistance programme in organic chemistry for a research fellowship to CSB. 


\section{References}

Born M 1920 Z. Phys. 145

Browı H C 1977 Nonclassical ion problem with comments by P V R Schleyer (New York: Plenum Press) Brown H C 1983 Acc. Chem. Res. 16 432, and references therein

Brown H C 1986 Acc. Chem. Res. 1934

Chiang J F, Wilcox C F and Bauer S H $1968 \mathrm{~J}$. Am. Chem. Soc. 903149

Cournoyer E M and Jorgensen W L $1984 \mathrm{~J}$. Am. Chem. Soc. 1065104

Dewar M J S, Haddon R C, Komornicki A and Rzepa H 1977 J. Am. Chem. Soc. 99377

Dewar M J S and Merz K M Jr 1986. J. Am. Chem. Soc. 1085634

Engstrom S, Jonsson B and Impey R W 1984 J. Chem. Phys. 805481

Goddard J D, Osamura Y and Schaefer III H F $1982 \mathrm{~J}$. Am. Chem. Soc. 1043258

Goering H L and Schewene C B $1965 \mathrm{~J}$. Am. Chem. Soc. 873516

Goetz D W, Schelegel H B and Allen L C $1977 \mathrm{~J}$. Am. Chem. Soc. 998118

Jorgensen W L and Munroe J E 1977 Tetrahedron Lett. 581

Klopman G $1969 \mathrm{~J}$. Am. Chem. Soc. 9189

Kohler H J and Lischka H $1979 \mathrm{~J}$. Am. Chem. Soc. 1013479

Myhre P C, McLaren K L, and Yannoni C S $1985 \mathrm{~J}$. Am. Chem. Soc. 1075294

Olah G A, Prakash G K S and Saunders M 1983 Acc. Chem. Res. 16440 , and references therein Raghavachari K, Haddon R C, Schleyer P V R and Schaefer H F III 1983 J. Am. Chem. Soc. 1055915 Rossky P J 1985 Ann. Rev. Phys. Chem. 36321

Tembe B L and McCammon J A 1984 Comput. Chem. 8281

Verlet L 1967 Phys. Rev. 15998

Wenke $G$ and Lenoir D 1979 Tetrahedron 35489

Winstein S and Trifan D $1952 \mathrm{~J}$. Am. Chem. Soc. 741147

Yannoni C S, Macho V and Myhre P C 1982 J. Am. Chem. Soc. 1047380.

Yoshimine M, McLean A D, Liu B, Defrees D J and Binkley J S 1983 J. Am. Chem. Soc. 1056185 